\title{
Original
}

\section{Mastectomía preservadora de piel con colgajo dermograso desepitelizado (técnica de Spira modificada) y reconstrucción mediante implante directo}

\author{
Marta Allué Cabañuz*, Maria Dolores Arribas del Amo, Ismael Gil Romea, \\ María Pilar Val-Carreres Rivera, Ramón Sousa Domínguez \\ y Antonio Tomás Güemes Sánchez
}

Unidad de Mama, Hospital Clínico Universitario Lozano Blesa, Zaragoza, España

INFORMACIÓN DEL ARTÍCULO

Historia del artículo:

Recibido el 7 de abril de 2020

Aceptado el 24 de abril de 2020

On-line el $\mathrm{xxx}$

Palabras clave:

Mastectomía preservadora de piel y pezón

Reconstrucción inmediata

Técnica de Spira

Complicaciones

Secuelas

\begin{abstract}
R E S U M E N
Introducción: La técnica de Spira es un tipo de mastectomía preservadora de piel que permite la reconstrucción inmediata (RMI), ideal en mamas ptósicas. Si bien, persiste controversia sobre resultados oncológicos en el cáncer de mama. El objetivo es analizar complicaciones, secuelas cosméticas, causas de reintervención y resultados oncológicos.

Métodos: Estudio observacional retrospectivo de pacientes intervenidas durante 2003-2018 en nuestro centro. La población de estudio la constituyen pacientes con carcinoma de mama o sometidas a mastectomía profiláctica por alto riesgo, en las que se realizó una mastectomía preservadora de piel con colgajo dermograso desepitelizado (técnica de Spira modificada) y reconstrucción mediante implante directo. Se analiza la presencia de complicaciones precoces y tardías, secuelas, recidiva tumoral y supervivencia.

Resultados: Se realizaron 247 mastectomías con reconstrucción en 139 pacientes, 216 bilaterales $(87,4 \%)$ y 31 unilaterales (12,5\%); 121 terapéuticas (49\%) y 126 profilácticas (51\%). La mediana de seguimiento fue de 81 meses. Se observaron complicaciones en un 16,2\%; necrosis cutánea en 5,3\% y cinco casos de necrosis del CAP (2\%). La tasa de reintervención por secuelas cosméticas fue del 17,4\% (la más frecuente fue contractura capsular 11,3\%) y, de ellas, el 39,3\% recibieron RT. La tasa de recidiva fue del 14\% (0,8\% cutánea, 3,3\% locorregional y $9,9 \%$ a distancia). Ocho pacientes fallecieron (6,6\%). La SLE y SG fue del $92,6 \%$ y $93,3 \%$ a cinco años.

Conclusión: La técnica de Spira constituye una opción segura y ofrece buenos resultados cosméticos y oncológicos como tratamiento y profilaxis de cáncer de mama en mamas ptósicas de moderado a gran tamaño.
\end{abstract}

(C) 2020 AEC. Publicado por Elsevier España, S.L.U. Todos los derechos reservados.

\footnotetext{
* Autor para correspondencia.

Correo electrónico: martitaallue@hotmail.com (M. Allué Cabañuz).

https://doi.org/10.1016/j.ciresp.2020.04.028

0009-739X/C 2020 AEC. Publicado por Elsevier España, S.L.U. Todos los derechos reservados.
} 
Keywords:

Nipple sparing mastectomy Immediate breast reconstruction Spira technique Complications Sequelae

\section{Nipple sparing mastectomy with a de-epithelialized derma-fat flap (modified Spira technique) and direct-to implant reconstruction}

\author{
A B S T R A C T
}

Introduction: Spira technique is a type of nipple-sparing mastectomy that allows immediate reconstruction (IBR), ideal for ptosic breasts. Although, controversy persists regarding oncological results in breast cancer. The aim is to analyze complications, cosmetic outcomes, causes of reoperation and oncological results.

Methods: Retrospective observational analysis of patients undergone surgery during 20032018 in our center. Study population is based on patients with breast carcinoma or undergoing prophylactic mastectomy due to high-risk, in which a skin-sparing mastectomy with a de-epithelialized derma-fat flap (modified Spira technique) and direct to implant reconstruction was performed. Short and long-term complications, sequelae, tumor recurrence and survival rates are analyzed.

Results: A total of 247 mastectomies with immediate reconstruction in 139 patients, 216 bilateral (87.4\%) and 31 unilateral (12.5\%) were performed. 121 therapeutic (49\%) and 126 prophylactic (51\%). Median follow-up 81 months. Complications were observed in $16.2 \%$; skin necrosis $5.3 \%$ and 5 cases of NAC necrosis (2\%). Reoperation rate due to cosmetic sequelae was $17.4 \%$ (capsular contracture was the most frequent,11.3\%) and a $39.3 \%$ of these patients have received RT. Recurrence of $14 \%(0.8 \%$ skin, $3.3 \%$ locoregional and $9.9 \%$ metastatic), 8 patients died (6.6\%). Rates of FSD and OS were $92.6 \%$ and $93.3 \%$ respectively.

Conclusion: Spira mastectomy is a safe option and provides good cosmetic and oncologic results as breast cancer treatment and prophylaxis in moderate-large ptosic breasts.

(C) 2020 AEC. Published by Elsevier España, S.L.U. All rights reserved.

\section{Introducción}

En 1977 Spira describió la mastectomía preservadora de piel utilizando el colgajo dermograso inferior ${ }^{1}$. Mediante esta técnica, enmarcada dentro de las mastectomías preservadoras de piel (o Skin Sparing Mastectomy tipo IV de la clasificación de Carlson), se consigue extirpar todo el tejido glandular incluyendo una pequeña porción de piel y todo el tejido retroareolar.

Habitualmente, es una técnica destinada a mamas de mediano o gran tamaño y con un grado de ptosis mínimo. La condición imprescindible es que el complejo areola-pezón (CAP) alcance al menos la altura del surco submamario, requisito fundamental para poder ejecutar esta técnica consiguiendo un resultado cosmético satisfactorio.

En esta técnica se extrae el CAP y se afina para después injertarlo en el nuevo montículo mamario creado. A pesar de que el autor no describe complicaciones en su experiencia inicial, a excepción de contractura capsular ${ }^{1}$, estudios recientes encuentran tasas generales de complicación en torno al $20 \%$, especialmente necrosis del CAP con posible pérdida del injerto ${ }^{2}$.

La principal ventaja de esta técnica es que nos permite realizar la reconstrucción en un solo tiempo quirúrgico, alojando el implante definitivo en posición parcialmente retromuscular y cubierto en su parte inferior por el colgajo dermograso, evitando la necesidad de utilizar matriz dérmica acelular y consiguiendo así un resultado cosmético favorable ${ }^{3}$, sin dejar de ser una técnica oncológicamente segura ${ }^{4} \mathrm{y}$ eficiente $^{5}$.

Mientras la mastectomía preservadora de piel está ampliamente validada como mastectomía terapéutica y no solo profiláctica $^{6}$, la preservación del CAP sigue generando controversia ${ }^{7}$, fundamentalmente en términos de recurrencia.

El objetivo del estudio es analizar las complicaciones postoperatorias, las secuelas cosméticas, las causas de reintervención y los resultados oncológicos de la mastectomía preservadora de piel con colgajo dermograso.

\section{Métodos}

\section{Selección de pacientes}

Pacientes sometidas a una mastectomía preservadora de piel con colgajo dermograso desepitelizado (técnica de Spira modificada) y reconstrucción mediante implante directo, operadas de manera consecutiva durante un período de 15 años (2003-2018) en una única unidad de mama. Se realiza un análisis observacional retrospectivo.

Se incluyen las siguientes afecciones y condiciones clínicas:

Pacientes con carcinoma de mama de novo.

Pacientes tratadas previamente por un cáncer de mama mediante cirugía conservadora que presentan recidiva o márgenes afectos y optan por la mastectomía en lugar de re-excisión. 
Mastectomía profiláctica contralateral en pacientes con un cáncer de mama (por factores de riesgo, elección de la paciente o búsqueda de simetría).

Mastectomía profiláctica bilateral en pacientes con mutaciones en BRCA $1 / 2$ o alto riesgo por historia familiar sin mutación demostrada.

Pacientes con un grado de ptosis mínimo (imprescindible que el CAP alcance al menos la altura del surco submamario).

Se excluyen los siguientes casos:

Carcinoma de mama inflamatorio

Reconstrucción mamaria en dos tiempos

Pacientes con metástasis a distancia al diagnóstico, puesto que no se les realiza la reconstrucción inmediata con implante.

Pacientes con mamas de pequeño tamaño o sin ptosis (se realizan otras técnicas).

\section{Técnica quirúrgica y tratamiento oncológico}

Fase de planificación

La planificación quirúrgica se realizó con las pacientes en posición de pie. En primer lugar, se realiza el marcaje cutáneo; se trata de un patrón en $\mathrm{V}$ invertida, se proyecta el surco inframamario sobre la línea mamaria media a $5 \mathrm{~cm}$ del CAP y a partir de la cual se trazan dos ramas verticales, intentando que sean lo más cerradas posible para preservar así la mayor cobertura cutánea y a partir de ellas trazamos las ramas horizontales hacia el surco inframamario (fig. 1).

Fase de mastectomía

La intervención se realiza con la paciente en posición semisentada. En primer lugar, se libera el CAP, pero no lo extirparemos hasta el final de la intervención (para así disminuir el tiempo de isquemia del injerto libre), después desepitelizamos el colgajo dermograso en el polo inferior de la mama. Se realiza la extirpación de la glándula mamaria preservando finos colgajos de piel según el patrón que hemos diseñado respetando un espesor mínimo de $1 \mathrm{~cm}$. Extirpamos el CAP conservándolo en una gasa humedecida con suero fisiológico y confeccionamos el colgajo dérmico. Por último, extirpamos la cola axilar de la mama y liberamos el musculo pectoral mayor del pectoral menor y de sus inserciones en la pared torácica para después cubrir el implante, también seccionamos el musculo pectoral mayor de sus inserciones mediales a nivel del esternón (fig. 1).

\section{Fase reconstructiva}

Reconstrucción en un solo tiempo mediante la colocación de un implante de silicona de perfil anatómico quedando doblemente cubierto mediante el colgajo dermograso desepitelizado suturado al músculo pectoral mayor. El volumen del implante depende del volumen de tejido mamario extraído y de la configuración de la mama afectada y la contralateral, individualizándose en cada caso. Por último, realizamos el cierre cutáneo siguiendo el patrón confeccionado, primero las ramas verticales sobre la proyección del surco inframamario en la línea media. Buscamos la zona de máxima proyección en el montículo creado a modo de nueva mama para marcar la zona donde realizaremos el injerto libre del CAP. En este momento comprobamos la viabilidad del mismo mediante examen visual y la ausencia de afectación del tejido retroareolar mediante biopsia intraoperatoria. Tras desepitelizar el área marcada y realizar pequeñas perforaciones en el injerto, suturamos el CAP mediante puntos sueltos de monofilamento no reaborsible de 4.0 y colocamos una gasa anudada presionando el injerto que se mantendrá durante una semana. Alojamos dos drenajes aspirativos, uno en la cámara del implante y otro a nivel subcutáneo, que retiramos cuando el débito es menor a 30 cc, muchas veces de forma ambulatoria (fig. 2).

La administración de quimioterapia (QT) o radioterapia (RT) se ajustó a las guías de práctica clínica del Centro en el momento del tratamiento.

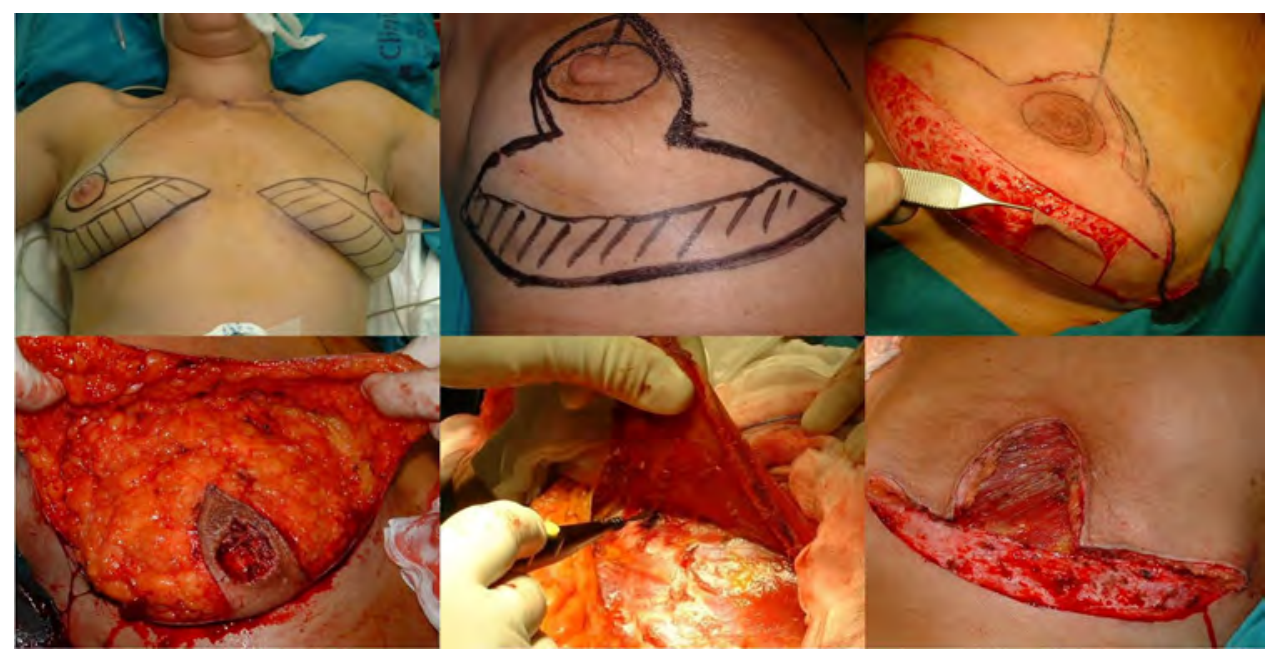

Figura 1 - Fase de mastectomía: a) Marcaje del patrón; b) Inclusión en el área a extirpar la cicatriz de la biopsia; c) Desepitelización del área que conformará el colgajo dermograso inferior; d) Extirpación y reserva del CAP; e) Mastectomía liberando adherencias al musculo; f) Resultado tras extirpación completa de la glándula. 


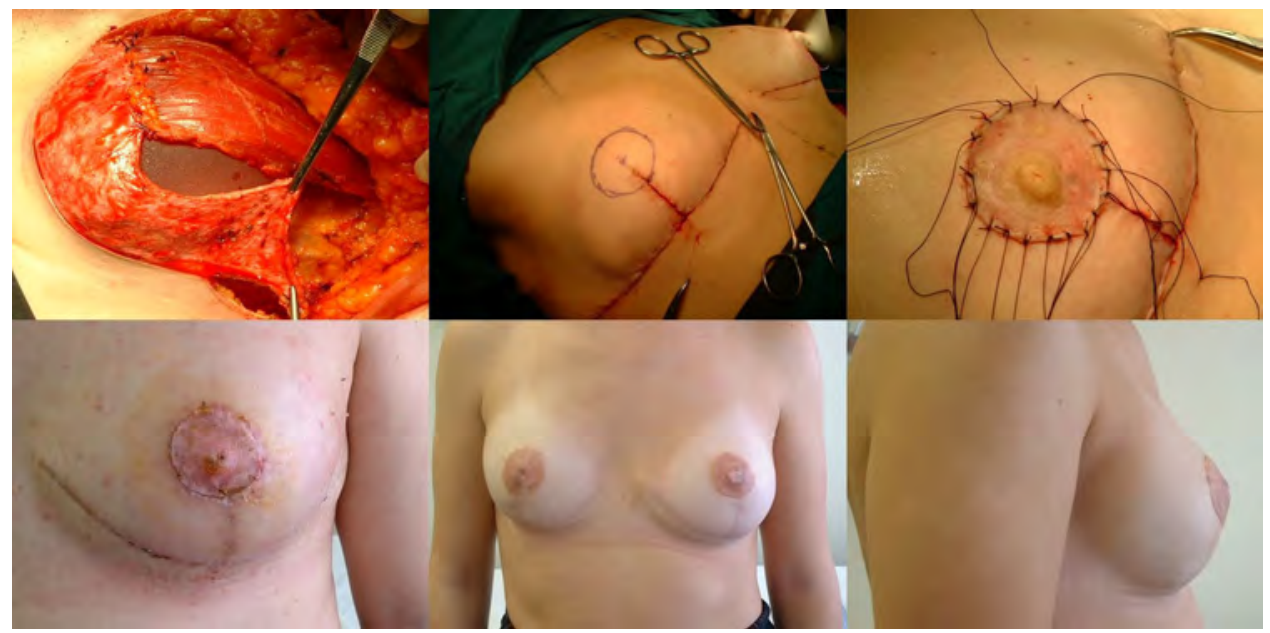

Figura 2 - Fase de reconstrucción: a) Colocación del implante que queda cubierto en los 2/3 superiores por musculo pectoral mayor, suturado al colgajo dermograso que cubre el 1/3 inferior; b) Marcaje del área del nuevo CAP una vez suturado el patrón cutáneo; c) injerto libre del CAP en su nueva posición; d) Resultado a los siete días; e) Resultado al año, visión frontal; f) Resultado al año, visión lateral.

\section{Variables de estudio}

Como variables de estudio, se diferencian cuatro grupos:

- Complicaciones: se valoran como tal la presencia de necrosis cutánea, diferenciando de forma específica la necrosis del CAP, que se definió como parcial, si consiguió recuperarse, y total, si requirió extirpación quirúrgica con reconstrucción secundaria, seroma mantenido, infección, hematoma.

- Reintervenciones: durante el primer mes se incluyen en el apartado de Complicaciones postoperatorias y pasado este tiempo son debidas a secuelas cosméticas.

- Secuelas cosméticas: se incluyen como tal la contractura capsular periprotésica, la rotura del implante, la extrusión protésica y pliegues cutáneos.

- Resultados oncológicos: la recurrencia locorregional se definió como aparición de nuevo tumor en la pared torácica ipsilateral (tejido subcutáneo y músculo pectoral) o recurrencia en la axila ipsilateral, ganglios linfáticos supraclaviculares, en cadenas mamarias internas o infraclaviculares. Se consideró la recurrencia exclusivamente cutánea a parte para el análisis. La recidiva a distancia (metástasis) fue definido como cualquier recurrencia en todas las demás áreas no incluidas en la recurrencia locorregional.

\section{Análisis estadístico}

Análisis descriptivo de las variables a estudio. Para variables cualitativas se aportan frecuencias relativas y absolutas. Para variables cuantitativas media y desviación estándar. Para el análisis se ha utilizado el programa estadístico SPSS 22.0 para Windows (SPSS Ibérica, Madrid, España).

Este estudio se adhiere a los principios éticos de la Declaración de Helsinki y fue aprobado por el comité ético del centro.

\section{Resultados}

\section{Descripción de la serie}

Durante el periodo de estudio se llevaron a cabo 247 mastectomías preservadoras de piel mediante técnica de Spira con reconstrucción inmediata en 139 pacientes. Del total, 216 fueron bilaterales $(87,4 \%)$ y 31 unilaterales $(12,5 \%)$. Se realizaron 121 mastectomías terapéuticas (49\%) [71 como tratamiento de un cáncer de novo $(28,7 \%)$ y 50 mastectomías sobre mamas tratadas previamente mediante cirugía conservadora (20,2\%)] y 126 mastectomías profilácticas (51\%) [92 mastectomías contralaterales profilácticas en pacientes con cáncer de mama $(37,2 \%)$ y 34 mastectomías reductoras de riesgo en pacientes de riesgo $(13,7 \%)]$. Las comorbilidades se exponen en tabla 1.

El 28\% recibió RT adyuvante posmastectomía, $24,7 \%$ habían recibido $\mathrm{RT}$ previamente y $47,1 \%$ de las mamas intervenidas no fueron radiadas. Sesenta y dos pacientes precisaron QT adyuvante (44,6\%), 27 habían recibido QT neoadyuvante $(19,4 \%)$ y 50 no recibieron tratamiento quimioterápico ni antes ni tras la intervención $(35,9 \%)$.

La QT adyuvante se administró una media de 47,3 días tras la intervención (DE 29,8) (rango 13-147). En cinco casos (5/62, $8 \%$ ) se demoró más de los 90 días que se estipulan por consenso como retraso, motivado en dos casos por infección postoperatoria y en uno por seroma mantenido. La mediana de seguimiento fue de 81 meses (DE 52,42).

\section{Complicaciones}

La tasa general de complicaciones fue del 16,2\% (40/247), siendo la necrosis cutánea la más frecuente $(13 / 247$ [5,3\%])

Solo se dieron cinco casos de necrosis del CAP tras injerto libre del mismo (2\%) y ocho pacientes precisaron reintervención en el postoperatorio a corto plazo (cinco casos por 


\section{Tabla 1 - Características pacientes}

\begin{tabular}{ll} 
Características de la población & N 247 (\%)* \\
\hline Indicación mastectomía & \\
Terapéutica & $121(49)$ \\
Por un cáncer de novo & $71(28,7)$ \\
Tras cirugía conservadora previa & $50(20,2)$ \\
Profiláctica & $126(51)$ \\
Profiláctica contralateral & $92(37,2)$ \\
Profiláctica bilateral & $34(13,7)$ \\
Edad media & $47,4 \pm 9,2$ años \\
Comorbilidades & \\
DM tipo 2 & $9(6,4)$ \\
IMC > 30 & $6(4,3)$ \\
Tabaquismo activo & $10(7,2)$ \\
Estadio tumoral & $\mathrm{N} 121(\%)^{* *}$ \\
CDis & $9(7,4)$ \\
I & $58(47,9)$ \\
II & $29(23,9)$ \\
III & $25(20,6)$ \\
Radioterapia (mastectomías terapéuticas) & $\mathrm{N} 121(\%)^{* *}$ \\
No & $57(47,1)$ \\
Anterior & $30(24,7)$ \\
Adyuvante & $34(28)$ \\
Quimioterapia & $\mathrm{N} 139$ pacientes \\
No & $50(35,9)$ \\
Neoadyuvante & $27(19,4)$ \\
Adyuvante (posmastectomía) & $62(44,6)$ \\
\hline &
\end{tabular}

* Tasas determinadas por número de mamas, excepto comorbilidades y quimioterapia, que fue determinado por número de pacientes.

* Tasas referidas al total de mastectomías terapéuticas.

sangrado y tres por infección, siendo estas las pacientes que posteriormente presentaron extrusiones protésicas que precisaron reintervención a largo plazo) (tabla 2).

\section{Secuelas cosméticas}

La tasa de secuelas cosméticas fue del 17,4\% (43/247), siendo la contractura capsular periprotésica grado III/IV la causa más frecuente de reintervención a largo plazo [28/247 (11,3\%)]. Del total de pacientes que presentaron contractura capsular, 11 habían recibido RT adyuvante [11/28 (39,3\%)] (tabla 3). La reintervención por secuelas se practicó con una media de 4,7 años $(\mathrm{DE} \pm 4,96)$ tras la intervención.

\section{Resultados oncológicos}

De las 121 mastectomías terapéuticas la tasa global de recidiva fue del 14\% (17/121), con un 0,8\% de recidiva cutánea, 3,3\% de

\section{Tabla 2 - Complicaciones}

\begin{tabular}{ll} 
Complicaciones & $40(16,2 \%)$ \\
\hline Necrosis cutánea & $13(5,3)$ \\
Seroma & $5(2,0)$ \\
Infección & $6(2,4)$ \\
Hematoma & $11(4,5)$ \\
Necrosis CAP & $5(2,0)$ \\
Reintervención en primer mes & $8(3,2)$ \\
\hline
\end{tabular}

Tabla 3 - Causas de reintervención a largo plazo por secuelas cosméticas

\begin{tabular}{ll} 
Secuelas cosméticas & $43(17,4 \%)$ \\
\hline Contractura capsular & $28(11,3)$ \\
Rotura implante & $8(3,2)$ \\
Extrusión protésica & $3(1,2)$ \\
Reconstrucción CAP & $2(0,8)$ \\
Pliegues cutáneos & $2(0,8)$ \\
\hline
\end{tabular}

recidiva locorregional y un 9,9\% de metástasis a distancia. No se observaron recidivas en el CAP.

Ocho pacientes fallecieron (6,6\%). El tiempo medio de supervivencia libre de enfermedad (SLE) fue de 5,08 años (DE 4,35 ) y el de supervivencia global (SG) de 5,78 años (DE 4,73). Las tasas de SLE y SG fueron 92,6\% y 93,3\% respectivamente. Los porcentajes por subgrupos se detallan en tabla 4.

\section{Discusión}

Debido al aumento en la detección de cánceres en etapas más tempranas la necesidad de técnicas de preservación de la piel ha aumentado en las últimas décadas de igual modo que la mastectomía contralateral profiláctica en pacientes con cáncer unilateral debido a la demanda de las pacientes ${ }^{8,9}$. Por otra parte, las pacientes demandan un resultado cosmético satisfactorio y eligen la reconstrucción mamaria en un solo acto quirúrgico si es posible ${ }^{10}$. Por todo ello, en muchas ocasiones, nos encontramos en la necesidad de realizar mastectomías bilaterales, preservando la piel y el CAP y que además permitan la colocación de un implante definitivo en el mismo acto quirúrgico. La técnica de Spira cubre a la perfección todas estas necesidades y resulta ideal en pacientes con mamas de mediano o gran tamaño y cierto grado de ptosis $^{11,12}$.

A diferencia de lo que ocurre con la mastectomía subcutánea en mamas pequeñas, obtener resultados óptimos en este tipo de mamas es más difícil y requiere reposicionar el CAP y disminuir la cobertura cutánea de la mama. La técnica, no exenta de riesgos, puede comprometer la cicatrización de heridas y la viabilidad del CAP lo que podría llevar a la necrosis cutánea con exposición del implante, incluso extrusión del mismo y fracaso de la reconstrucción ${ }^{13,14}$. Se han propuesto dos mecanismos que explicarían estos problemas de cicatrización/perfusión; por un lado, colgajos dérmicos demasiado largos y por otro, que estos sean muy finos, poniendo así en peligro su vascularización a través del plexo subdérmico ${ }^{15}$.

En nuestra experiencia encontramos una incidencia de necrosis cutánea del 5,3\%, necrosis del CAP del 2\% y necesidad de reinterveción por extrusión protésica en tres pacientes $(1,2 \%)$, tasas inferiores a las reportadas en otras series, como la de King et al. que presenta un $10 \%$ de necrosis cutánea ${ }^{16}$.

Por otra parte, varios estudios han demostrado que la tasa de recurrencia local promedio después de mastectomías preservadoras de piel no es diferente de la informada cuando se conserva o no el CAP (3,9 vs. $3,25 \%$, respectivamente) ${ }^{17}$. Además, existe evidencia de que combinar estas técnicas con una reconstrucción inmediata no retrasa significativamente la administración de terapia adyuvante ${ }^{18}$. Nuestra serie apoya 
Tabla 4 - Resultados oncológicos en casos terapéuticos

\begin{tabular}{|c|c|c|c|c|c|c|}
\hline & $\mathrm{n}$ & $\begin{array}{l}\text { Recidiva } \\
\text { cutánea } \\
\text { n (\%) }\end{array}$ & $\begin{array}{l}\text { Recurrencia } \\
\text { locorregional } \\
\mathrm{n}(\%)\end{array}$ & $\begin{array}{l}\text { Recidiva a } \\
\text { distancia } \\
\mathrm{n}(\%)\end{array}$ & $\begin{array}{l}\text { Exitus } \\
\mathrm{n}(\%)\end{array}$ & $\begin{array}{l}\text { No recidiva } \\
\mathrm{n}(\%)\end{array}$ \\
\hline Total mastectomías terapéuticas & 121 & $1(0,8)$ & $4(3,3)$ & $12(9,9)$ & $8(6,6)$ & $96(79,3)$ \\
\hline Cáncer de novo & 71 & $1(1,4)$ & $3(4,2)$ & $7(9,8)$ & $3(4,2)$ & $57(80,3)$ \\
\hline CDis & 4 & 0 & 0 & $1(25)$ & $1(25)$ & $2(50)$ \\
\hline $\begin{array}{l}\text { Carcinoma } \\
\text { invasivo }\end{array}$ & 67 & $1(1,4)$ & $3(4,4)$ & $6(8,9)$ & $2(2,9)$ & $55(82,1)$ \\
\hline Tratadas previamente & 50 & 0 & $1(2)$ & $5(10)$ & $5(10)$ & $39(78)$ \\
\hline CDis & 5 & 0 & 0 & $1(20)$ & $1(20)$ & $3(60)$ \\
\hline $\begin{array}{l}\text { Carcinoma } \\
\text { invasivo }\end{array}$ & 45 & 0 & $1(2,2)$ & $4(8,8)$ & $4(8,8)$ & $36(80)$ \\
\hline
\end{tabular}

estos datos, con un 0,8\% de recidiva cutánea, 3,3\% de recidiva locorregional y ningún caso de recidiva en CAP. Además, solo observamos retraso significativo (mayor de 90 días) en la administración de quimioterapia adyuvante en cinco casos, dos por infección postoperatoria y en uno por seroma mantenido.

A pesar de que el presente trabajo cuenta con un número considerable de casos y un periodo de seguimiento amplio presenta limitaciones al tratarse de un análisis retrospectivo con la experiencia de una única institución, por lo que no podemos generalizar los resultados.

En conclusión, la mastectomía preservadora de piel con colgajo dermograso desepitelizado y reconstrucción mediante implante directo constituye una opción segura y ofrece buenos resultados cosméticos y oncológicos en el tratamiento y profilaxis del cáncer de mama para pacientes con mamas ptósicas de moderado a gran tamaño.

\section{Financiación}

El trabajo no ha recibido financiación

\section{Conflicto de intereses}

Los autores declaran no tener ningún conflicto de intereses.

\section{Agradecimientos}

A los integrantes de Unidad de Mama del Hospital Clínico Universitario Lozano Blesa de Zaragoza, cirujanos, personal de enfermería y a las pacientes.

\section{B I B L I O G R A F Í A}

1. Spira M. Subcutaneous mastectomy in the large ptotic breast. Plast Reconstr Surg. 1977;59:200-5.

2. Jepsen C, Hallberg H, Pivodic A, Elander A, Hansson E. Complications, patient-reported outcomes, and aesthetic results in immediate breast reconstruction with a dermal sling: A systematic review and meta-analysis. J Plast
Reconstr Aesthet Surg. 2019;72:369-80. http://dx.doi.org/ 10.1016/j.bjps.2018.12.046.

3. Torstenson T, Boughey JC, Saint-Cyr M. Inferior dermal flap in immediate breast reconstruction. Ann Surg Oncol. 2013;20:3349. http://dx.doi.org/10.1245/s10434-013-3109-0.

4. Domergue S, Ziade M, Lefevre M, Prud'homme A, Yachouh J. Dermal flaps in breast reduction: prospective study in 100 breasts. J Plast Reconstr Aesthet Surg. 2014;67:e147-50. http://dx.doi.org/10.1016/j.bjps.2014.02.004.

5. Poruk KE, Ying J, Chidester JR, Olson JR, Matsen CB, Neumayer L, et al. Breast cancer recurrence after nipplesparing mastectomy: one institution's experience. Am J Surg. 2015;209:212-7. http://dx.doi.org/10.1016/ j.amjsurg.2014.04.001.

6. Garwood ER, Moore D, Ewing C, Hwang ES, Alvarado M, Foster $\mathrm{RD}$, et al. Total skin-sparing mastectomy: complications and local recurrence rates in 2 cohorts of patients. Ann Surg. 2009;249:26-32. http://dx.doi.org/ 10.1097/SLA.0b013e31818e41a7.

7. Ziogas D, Roukos DH, Zografos GC. Nipple-sparing mastectomy: overcoming oncological outcomes challenges. Ann Surg Oncol. 2010;17:323-4. http://dx.doi.org/10.1245/ s10434-009-0689-9.

8. Tuttle TM, Habermann EB, Grund EH, Morris TJ, Virnig BA. Increasing use of contralateral prophylactic mastectomy for breast cancer patients: a trend toward more aggressive surgical treatment. J Clin Oncol. 2007;25:5203-9. http:// dx.doi.org/10.1200/JCO.2007.12.3141.

9. Jones NB, Wilson J, Kotur L, Stephens J, Farrar WB, Agnese DM. Contralateral prophylactic mastectomy for unilateral breast cancer: an increasing trend at a single institution. Ann Surg Oncol. 2009;16:2691-6. http://dx.doi.org/10.1245/ s10434-009-0547-9.

10. Reza Goyanes M, Andradas Aragonés E, Blasco Amaro JA. Reconstrucción mamaria inmediata. Revisión sistemática y evaluación de resultados en una unidad de RMI de la Comunidad de Madrid. Madrid: Unidad de Evaluación de Tecnologías Sanitarias (UETS), Agencia Laín Entralgo. 2005. Disponible en: https://www.comunidad.madrid/sites/ default/files/aud/sanidad/ reconstruccion_mamaria_inmediata.2005_0.pdf.

11. Munhoz AM, Montag E, Filassi JR, Gemperli R. Immediate nipple-areola-sparing mastectomy reconstruction: An update on oncological and reconstruction techniques. World J Clin Oncol. 2014;5:478-94. http://dx.doi.org/10.5306/ wjco.v5.i3.478.

12. Spear SL, Willey SC, Feldman ED, Cocilovo C, Sidawy M, AlAttar A, et al. Nipple-sparing mastectomy for prophylactic and therapeutic indications. Plast Reconstr Surg. 2011;128:1005-14. http://dx.doi.org/10.1097/ PRS.0b013e31822b6456. 
13. Rusby JE, Brachtel EF, Michaelson JS, Koerner FC, Smith BL. Breast duct anatomy in the human nipple: threedimensional patterns and clinical implications. Breast Cancer Res Treat. 2007;106:171-9. http://dx.doi.org/10.1007/ s10549-006-9487-2.

14. Crowe JP, Patrick RJ, Yetman RJ, Djohan R. Nipple-sparing mastectomy update: one hundred forty-nine procedures and clinical outcomes. Arch Surg. 2008;143:1106-10. http:// dx.doi.org/10.1001/archsurg.143.11.1106.

15. Radovanovic Z, Radovanovic D, Golubovic A, Ivkovic-Kapicl $\mathrm{T}$, Bokorov B, Mandic A. Early complications after nipplesparing mastectomy and immediate breast reconstruction with silicone prosthesis: results of 214 procedures. Scand J Surg. 2010;99:115-8. http://dx.doi.org/10.1177/ 145749691009900302.
16. King IC, Harvey JR, Bhaskar P. One-stage breast reconstruction using the inferior dermal flap, implant, and free nipple graft. Aesthetic Plast Surg. 2014;38:358-64. http:// dx.doi.org/10.1007/s00266-014-0276-8.

17. Rivadeneira DE, Simmons RM, Fish SK, Gayle L, La Trenta GS, Swistel A, et al. Skin-sparing mastectomy with immediate breast reconstruction: a critical analysis of local recurrence. Cancer J. 2000;6:331-5.

18. Kahn J, Barrett S, Forte C, Stallard S, Weiler-Mithoff E, Doughty JC, et al. Oncoplastic breast conservation does not lead to a delay in the commencement of adjuvant chemotherapy in breast cancer patients. Eur J Surg Oncol. 2013;39:887-91. http://dx.doi.org/10.1016/j.ejso.2013.05.005. 